\title{
Dynamic Signature Recognition Based on Fisher Discriminant
}

\author{
Teodoro Schmidt, Vladimir Riffo, and Domingo Mery \\ Pontificia Universidad Catolica de Chile, PUC \\ \{theo,vriffo1\}@uc.cl, dmery@ing.puc.cl
}

\begin{abstract}
Biometric technologies are the primary tools for certifying identity of individuals. But cost of sensing hardware plus degree of physical invasion required to obtain reasonable success are considered major drawbacks. Nevertheless, the signature is generally accepted as one means of identification. We present an approach on signature recognition using face recognition algorithms to obtain class descriptors and then use a simple classifier to recognize signatures. We also present an algorithm to store the writing direction of a signature, applying a linear transformation to encode this data as a gray scale pattern into the image. The signatures are processed applying Principal Components Analysis and Linear Discriminant Analysis creating descriptors that can be identified using a $K N N$ classifier. Results revealed an accuracy performance rate of $97.47 \%$ under cross-validation over binary images and an improvement of $98.60 \%$ of accuracy by encoding simulated dynamic parameters. The encoding of real dynamic data boosted the performance rate from $90.21 \%$ to $94.70 \%$ showing that this technique can be a serious contender to other signature recognition methods.
\end{abstract}

Keywords: signature recognition, on-line signatures, off-line signatures, fishersignatures.

\section{Introduction}

In modern world trust between individuals has become a key factor in every activity. This enforces the need of authentication for all individuals involved in any given transaction. To accomplish the latter, biometric recognition employs two strategies: physical based characteristics and behavioral based characteristics 1. Within the latter, the signature outstands for its social acceptance and relatively low implementation costs 2]. Even legal regulations on most countries accept signature as a key discriminant factor. Hence, correct signature identification is crucial to guarantee the suitability of any transaction taking place. This paper presents a signature's analysis technique to determine whether or not it belongs to a given person, analyzing the signature's image against the results of a previous training process. Given its importance, signatures are subject to counterfeiting. Against this, the automatic signature recognition faces two main problems: the need to identify intrinsic static characteristics of the 
signature in question, such as its geometry (process known as off-line), and the need to identify graphological characteristics of the individual's signature, such as unique patterns of hand movements, speed and direction of writing, known as on-line analysis [3. Thus, the problem of identifying people lies in finding efficient algorithms to analyze static and dynamic signature characteristics, and then compare those analyses results in real time against a knowledge base of signatures, previously generated. This document is organized as follows: section II describes the state of the art of signatures recognition. Section III describes the proposed method based on principal component analysis (PCA) and linear discriminant analysis (LDA). This section also details the equations used to represent the signature's writing direction. Section IV presents the experimental development, including results analysis. Finally, Section V presents conclusions and scope of this paper plus future work of this research.

\section{Related Work}

The two most common approaches current investigations explore are: signature changes analysis in time domain and shape analysis of signature stroke morphology. Relevant works on the first approach are [4, 5] where temporal signature evolution is analyzed using multi-section vector quantization. On the second approach, work [6] analyzes gravity, eccentricity, skewness, with good accuracy results. Ad hoc selection of features can be used to increase accuracy 7]. This concept is extended by sub pattern analysis of signature's stroke [8] and the analysis of humans' perception of stroke segments 9. An issue here is the amount of data to be analyzed. One approach is to reduce the dimensionality of the feature space while maintaining discrimination between classes. A relevant work is [10] where LDA is used for dimensionality reduction and Neural Networks for classification. The drawback is that NN are hard to conceptualize due to their black box nature 11. Nonetheless, as the potential of dimensionality reduction is obvious, a recognition method should have a simpler classifier and better feature extraction. A special note deserves the idea in [12] where a color scheme is used, based on signature changes. This creates a unique color-based fingerprint for every signature, though these fingerprints are based on morphology changes rather than dynamic features. Our method uses dimensionality reduction as face recognition methods do, that is, by using PCA [13] and LDA to create feature vectors like EigenFaces [14], and FisherFaces [15], and a simple $K N N$ algorithm as classifier. We strengthen the capture process by creating a gray scale color based algorithm to encode dynamic features on to signature images.

\section{Proposed Method}

The action of signing is unique and exclusive for each individual. This is based not only in its geometry but on the existence of characteristics of the signature process itself, such as speed and direction of the signing action [16. Given this, it 
is very difficult to replicate the static characteristics [17] and dynamic characteristics of another individual's signature, without committing errors in the process. The hypothesis that it is possible to recognize the subject issuer of a signature using algorithms that belong to the face recognition problem [18 opens the possibility of using dynamic characteristics to encode extra information within the signatures images while capturing them. Nonetheless, the feature extraction process can theoretically be also applied to static characteristics. Based on the latter, our model proposes static analysis of vector of characteristics specific to signatures captured off-line, creating Fishersignatures, which correspond to principal component analysis and linear discriminator applied over the images. The whole recognition process is divided in two sections: i) training using Fishersignatures method over a set of images, and ii) testing using a new image as input for comparison against the already trained matrix of weights resulting from the section i). Additionally, we propose an algorithm to acquire dynamic characteristics when capturing the signatures. This method encodes the data into the original signature image, strengthening the features extraction process. The complete signature recognition system used is shown schematically in Figure 1.

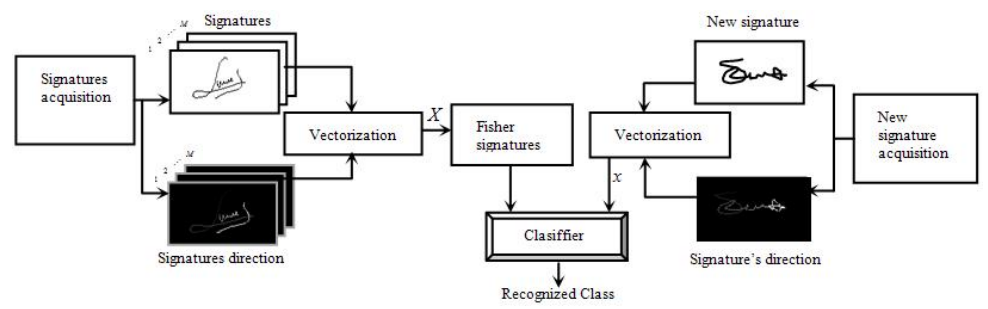

Fig. 1. Block diagram of the system proposed

\subsection{Fishersignatures Training Method}

Our technique for signatures recognition is based on the Eigenfaces matrix used in face recognition to project images onto a lower dimensional space, reducing computational complexity of features extraction. Given a set of signature images per class $\left\{I_{j}(x, y), j=1,2, \ldots, M\right\}$, being $I_{j}$ a matrix of order $N=m \mathrm{x} n$, the images are column-stacked vectorized (rasterized) and named $x_{j}, j=1,2, M$.

The vectorized training set is $X=\left[X_{1} X_{2} \ldots X_{c}\right]$ with $X_{k}=\left[x_{1}^{k} x_{2}^{k} \ldots x_{M}^{k}\right]$, $k=1,2, \ldots, c$, where $x_{j}^{k}$ is the vectorized image $j$ for class $k$. The order of $X$ is $N \times D$, with $D=M \times c$

The inter-class average of the images is a vector of $N$ elements:

$$
\mu_{k}=\frac{1}{M} \sum_{j=1}^{M} x_{j}^{k} \quad, k=1,2, \ldots, c
$$


The class average is a vector with $N$ elements:

$$
\mu=\frac{1}{(M \times c)} \sum_{k=1}^{c} \sum_{j}^{M} x_{j}^{k}
$$

The difference between each image and the class average is $A=\left[A_{1} A_{2} \ldots A_{D}\right]$ where $A_{d}$, with $d=1,2, \ldots, D$, are in turn:

$$
A_{d}=x_{j}^{k}-\mu \quad, d=1,2, \ldots, D
$$

The covariance matrix is defined as:

$$
S_{T}=A A^{T}
$$

Next is the calculation of the Eigen vectors of $A A^{T}$, defined as $u_{i}$. The trick here is to find the $v_{i}$ Eigen vectors of a new matrix $A^{T} A$, with $\lambda_{i}$ being the Eigen vectors of both $A A^{T}$ and $A^{T} A$, related through the following equality:

$$
u_{i}=A v_{i}
$$

The search for the $v_{i}$ Eigen vectors is carried out using the Jacobi method [19], where all $v_{i}$ are placed in descending order, following the order of the Eigen values $\lambda_{i}$. After normalizing $\left\|u_{i}\right\|=1$, all $u_{i}$ Eigen vectors are concatenated to form a $U$ matrix of order $N \times D$, where $U=\left[u_{1} u_{2} \ldots u_{i}\right], i=1,2, \ldots, D$. Finally, the $W_{E}$ projection matrix gets defined as:

$$
W_{E}=U^{T} A
$$

Fisher discriminant increases the separation between classes preserving a low discrimination inside every class. Fisher is considered an implementation of LDA over PCA space. With this, the dimensionality of $U$ can be reduced to $N \times D_{p}$, with $D_{p}=(M \cdot c)-c$, by redefining $U$ as a new matrix $W_{p c a}$. The new data projection on the reduced PCA space gets defined by $W_{E F}$ of order $D_{p} \mathrm{x} D$ :

$$
W_{E F}=W_{p c a}^{T} X
$$

More in detail, $W_{E F}=\left[w_{1}^{k} w_{2}^{k} \ldots w_{M}^{k}\right]$. The above reduction redefines the class average with a new equation where $w_{j}^{k}$ is the $j$ projected vectorized image of class $k$ :

$$
\eta_{k}=\frac{1}{M} \sum_{j=1}^{M} w_{j}^{k} \quad, k=1,2, \ldots, c
$$

Following the above transformation, the new equation for the inter-class average is:

$$
\eta=\frac{1}{(M \times c)} \sum_{k=1}^{c} \sum_{j}^{M} w_{j}^{k}
$$


In the same way, the class dispersion matrix gets determined by:

$$
S_{B}=\sum_{k=1}^{c}\left(\eta_{k}-\eta\right)\left(\eta_{k}-\eta\right)^{T}
$$

And the inter-class dispersion matrix gets determined by:

$$
S_{W}=\sum_{k=1}^{c} \sum_{j=1}^{M}\left(w_{j}^{k}-\eta\right)\left(w_{j}^{k}-\eta\right)^{T}
$$

It's interesting to note that $S_{B}$ and $S_{W}$ are square matrices of order $D_{p} \mathrm{x} D_{p}$. In order to ensure that $S_{B}$ and $S_{W}$ are related by $S_{B} W_{f l d}=S_{W} W_{f l d} \lambda$, the $W_{f l d}$ Eigen vectors and $\lambda$ Eigen values are calculated defining what we call Fishersignatures, with the following equation:

$$
P=W_{p c a} W_{f l d}
$$

Finally, the new $W_{E}$ projection matrix of Fishersignatures gets defined as:

$$
W_{E}=P^{T} A
$$

\subsection{Testing Method}

To classify a new signature, a $K N N$ search against the closest neighbor is performed, with the following steps:

a.- Testing signature $I$ is vectorized in to vector $x$ of order $N \times 1$ with $N=m \times n$

b.- Inter-class average $O$ is obtained from equation $O=x-\mu$

c.- LDA projection $W_{P}$ is carried out using $P$ and $O$ : $W_{P}=P^{T} O$

d.- Euclidean distance from $W_{E}$ to $W_{P}$ denotes a distance vector $\sqrt{\sum\left|W_{E}-W_{P}\right|^{2}}$ in which the lowest value corresponds to the signature's identified class.

\subsection{Signature's Writing Direction Encoding Method}

In order to capture dynamic information, such as the signature's writing direction, a data encoding method was developed. This method strengthens the feature extraction process by visually encoding extra information into the image, at capture time. A gray value is assigned to each pixel of the signature's track being captured. The background of the captured image is set to zero to give more contrast. The gray value for first pixel $t_{1}$ of the signature's track is 0.1 , to distinguish it from the background. The gray value for last pixel of the signature's track is 1 .

Let $T(x, y)=t_{1}\left(x_{1}, y_{1}\right), t_{2}\left(x_{2}, y_{2}\right), \ldots, t_{i}\left(x_{i}, y_{i}\right), \ldots, t_{n}\left(x_{n}, y_{n}\right)$ be a Cartesian coordinates vector representing the signature's track, with $t_{1}\left(x_{1}, y_{1}\right)$ being the first pixel written, and $t_{n}\left(x_{n}, y_{n}\right)$ being the last written. Each $t_{i}$ pixel of vector $T$ is assigned a gray level value given by the linear equation: 


$$
t_{i}=0.9 \frac{i-1}{n-1}+0.1
$$

The background of binary captured signatures is usually set to 1 and signature's track to 0 , but the above transformation captures the signature's track with a black-to-white gradient denoting the direction in which the signature was written, starting from pixel $t_{1}$ (lowest gray value), to last pixel $t_{n}$ (highest value). This effect is shown in Figure 2
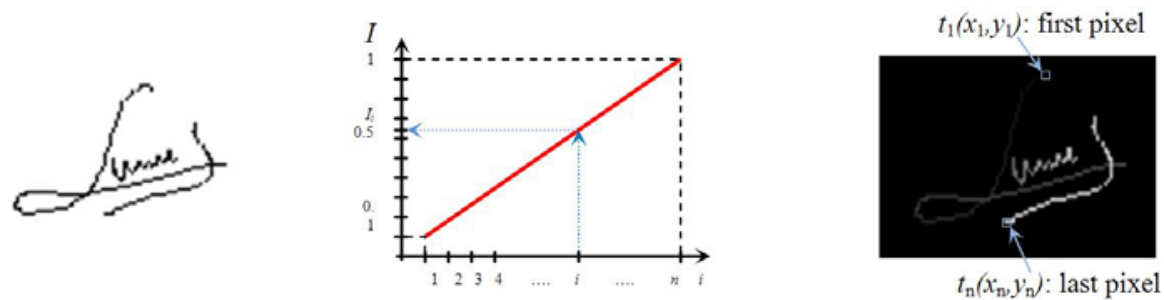

Fig. 2. Binary captured signature (left). Transformation to encode direction of signature (center). Result of visually encoded direction (right).

Simple visual inspection clearly shows that the image containing the signature's direction encoded in gray scale delivers more information than the binary one, even though they both share the same geometrical information, hence a Fishersignatures training and classification process using these gray scale images should deliver better performance results than their corresponding binary counterparts.

\section{Experiments and Results}

The database used for this work was GPDS960signature [20, with 960 classes, 24 images per class, in variable sizes. All images were normalized and resized to $102 \times 64$ pixels. These values come from the size of a tablet device used in a previous work to create a custom signature $\mathrm{db}$. We preserved the resolution for comparison reasons.

Our implementation of Eigen values and vectors search rely on singular value decomposition, requiring a lot of RAM for big matrices. To solve this issue, the algorithms were tested over a smaller data set, split in 3 groups, keeping 20 signatures per class in each group: one set with 100 classes; another set with 200 classes; and a third set with 300 classes. No counterfeit signatures were used as the nature of this work was to verify performance of Fishersignatures idea using cross-validation. These signatures were not originally captured using the encoding process proposed in section 3.3. In order to verify the strengthening capability of such an algorithm, writing direction simulations were applied over the original b/w images. The accuracy performance of the original Fishersignatures classification (created with the original b/w images) was compared to new 
Fishersignatures classification (created with simulated writing direction encoded onto the same images). Four different writing direction simulations were applied to each of the 3 data sets: first $40 \%$ of the images of a data set were applied a black-to-white (gray) gradient from left to right. Next $20 \%$ of the images of the same data set were applied the gradient from right to left. Next $20 \%$ of the images of the same data set were applied a top-down gradient. Final $20 \%$ of the images of the same data set were applied a bottom-up gray gradient. These percentages were arbitrarily chosen, based on the fact that people in western countries write from left to right, hence, simulation of this direction takes the biggest proportion. All other simulations equally share the remaining $60 \%$. In order to maintain simplicity, the classifier used for all tests was $K N N$ matching the first neighbor found for each class.
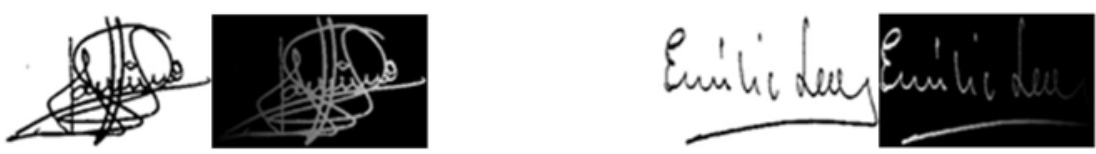

Fig. 3. Examples of simulated writing direction using a black-to-white gradient. Binary captured signature and left-to-right direction simulation (left). Binary captured signature and right-to-left direction simulation (right).

Performance results were evaluated through stratified cross validation using $5 \%$ of the data to test and the remaining $95 \%$ for training. Stratification ensures the representation of each class in the test sets. The overall performance of the method proposed is the average of 20 performances obtained. The average performance is shown in Table 1 .

To fully test the proposed data encoding algorithm, a second experiment was executed. This time, the writing direction (dynamic data) was encoded in real time during the acquisition process. The resulting db is SRM-SDB [18 with 45 classes, 10 signatures per class, and all images acquired using the method described in 3.3 (each signature's writing direction encoded in gray scale). A $\mathrm{b} / \mathrm{w}$ version of the images was also created for later use, where signature track's

Table 1. Accuracy performance results using cross-validation over 3 sets of images. Tests were carried out twice over each data set, one over binary images, and the next run over images with an encoded writing direction simulation.

\begin{tabular}{llc}
\hline \hline Data Set & Image type & Accuracy \% \\
\hline 100 individuals & Binary & $92.20 \%$ \\
100 individuals & Encoded simulation & $95.15 \%$ \\
200 individuals & Binary & $97.00 \%$ \\
200 individuals & Encoded simulation & $97.58 \%$ \\
300 individuals & Binary & $97.47 \%$ \\
300 individuals & Encoded simulation & $98.60 \%$ \\
\hline
\end{tabular}


Table 2. Accuracy performance results using cross-validation over signatures with real writing direction data encoded in gray scale and binary versions of same images

\begin{tabular}{llc}
\hline \hline Data Set & Image type & Accuracy \% \\
\hline 45 individuals & Binary (no gradient) & $90.21 \%$ \\
45 individuals & Encoded real writing direction & $94.70 \%$ \\
\hline
\end{tabular}

gray values were replaced by 0 (black) and background values were replaces by 255 (white). The accuracy of Fishersignatures created using the original gray scale acquired images was compared to Fishersignatures created using binarized images. The classifier was $K N N$ matching the first neighbor found per class. Performance results were evaluated using stratified cross validation with $10 \%$ of data to test and $90 \%$ for training. The average performance is shown in Table 2 .

\section{Conclusions}

In this paper we propose two contributions for an improved signature recognition technique: One contribution is the implementation of Fisher discriminant based feature vectors, we called Fishersignatures, a la face recognition method. The second contribution is our feature strengthening method of encoding dynamic parameters while acquiring signatures, particularly the signature's writing direction.

The first contribution shows that our Fishersignatures implementation creates good class separation. Even if applied over black and white images, the use of a simple classifier, such as $K N N$, to identify signatures delivers an accuracy of $97.47 \%$ in the best $\mathrm{b} / \mathrm{w}$ case.

The second contribution shows that the signature acquisition process can be greatly improved by encoding extra information into a signature, without modifying its morphological characteristics, and still allow the processing of images using Fishersignatures plus a simple $K N N$ classifier. This statement gets validated by two different successful experiments:

I) Encoding of simulated writing direction over binary-acquired signatures: the best accuracy rate achieved under binary analysis (97.47\%) was superseded by an accuracy of $98.60 \%$ when encoding simulated dynamic information into the images.

II) Encoding of real writing direction at acquisition time: the proposed encoding method tested in a real-life scenario delivered an accuracy rate of $94.70 \%$, which is far superior than $90.21 \%$ of accuracy obtained using a $\mathrm{b} / \mathrm{w}$ version of the same images.

Although both experiments are obviously not comparable between them (given the nature of data acquisition of each experiment plus number of classes, samples, folds, etc.), it can be observed that Fishersignatures classification always delivered an accuracy of over $90 \%$ in all cases, and also that the proposed encoding method raised this accuracy in both experiments. The accuracy rate of other 
techniques is: $93 \%$ obtained by Karouni et al. [6], 94\% obtained by Al-Mayyan et al. when using PCA [7], 93\% obtained by Radhika et al. 8]. A further comparison of the best accuracy performance obtained in the first experiment $(98.60 \%)$ against these other techniques shows that Fishersignatures classification delivers excellent performance, even though the $K N N$ classifier seems weaker than others. Finally, accuracy results obtained denote that the combination of our two contributions can become a serious contender to other signature recognition methods.

An extension of the encoding algorithm is planned for future work, where other dynamic parameters will be encoded, such as writing speed. The replacement of the classifier for a stronger one, plus the analysis of a higher volume of signatures are also in our research roadmap.

Acknowledgments. This work was supported in part by School of Engineering, Pontificia Universidad Catolica de Chile, Grant FIA.

\section{References}

1. Jain, A.K., Ross, A., Prabhakar, S.: An introduction to biometric recognition. IEEE Transactions on Circuits and Systems for Video Technology 14, 4-20 (2004)

2. Prabhakar, S., Pankanti, S., Jain, A.K.: Biometric recognition: security and privacy concerns. IEEE Security \& Privacy 1, 33-42 (2003)

3. Faundez-Zanuy, M.: On-line signature recognition based on VQ-DTW. Pattern Recognition 40, 981-992 (2007)

4. Pascual-Gaspar, J.M., Faundez-Zanuy, M., Vivaracho, C.: Fast on-line signature recognition based on VQ with time modeling. Engineering Applications of Artificial Intelligence 24(2), 368-377 (2011)

5. Pascual-Gaspar, J.M., Faundez-Zanuy, M., Vivaracho, C.: Efficient on-line signature recognition based on multi-section vector quantization. Pattern Analysis \& Applications 14(1), 37-45 (2010)

6. Karouni, A., Daya, B., Bahlak, S.: Offline signature recognition using neural networks approach. In: Procedia Computer Science, World Conference on Information Technology, vol. 3, pp. 155-161 (2011)

7. Al-Mayyan, W., Own, H.S., Zedan, H.: Rough set approach to online signature identification. Digital Signal Processing 21(3), 477-485 (2011)

8. Radhika, K.R., Venkatesha, M.K., Sekhar, G.N.: Signature authentication based on subpattern analysis. Applied Soft Computing 11(3), 3218-3228 (2011)

9. Ebrahimpour, R, Amiri, A., Nazari, M., Hajiany, A.: Robust Model for Signature Recognition Based on Biological Inspired Features. International Journal of Computer and Electrical Engineering 2(4) (August 2010)

10. Meshoul, S., Batouche, M.: A novel approach for online signature verification using fisher based probabilistic neural networks. In: Proceedings - IEEE Symposium on Computers and Communications, pp. 314-319 (2010)

11. Tu, J.V.: Advantages and disadvantages of using artificial neural networks versus logistic regression for predicting medical outcomes. Journal of Clinical Epidemiology 49(11), 1225-1231 (1996)

12. Kulkarni, V.B.: A Colour Code Algorithm for Signature Recognition. Electronic Letters on Computer Vision and Image Analysis 6, 1-12 (2007) 
13. Turk, M., Pentland, A.: Eigenfaces for Recognition. Journal of Cognitive Neuroscience 3, 71-86 (1991)

14. Turk, M., Pentland, A.: Face recognition using eigenfaces. In: Proceedings of Computer Vision \& Pattern Recognition, CVPR 1991, IEEE Computer Society Conference, pp. 586-591 (1991)

15. Belhumeur, P., Hespanha, J., Kriegman, D.: Eigenfaces vs. Fisherfaces: recognition using class specific linear projection. IEEE Transactions on Pattern Analysis and Machine Intelligence 19, 711-720 (1997)

16. Vivaracho-Pascual, C., Faundez-Zanuy, M., Pascual, J.M.: An efficient low cost approach for on-line signature recognition based on length normalization and fractional distances. Pattern Recognition 42, 183-193 (2009)

17. Erkmen, B., Kahraman, N., Vural, R., Yildirim, T.: CSFNN optimization of signature recognition problem for a special VLSI NN chip. In: 3rd International Symposium on Communications, Control and Signal Processing, ISCCSP 2008, pp. 1082-1085 (2008)

18. Riffo, V., Schmidt, T., Mery, D.: Propuesta Novedosa de Reconocimiento Dinmico de Firmas. In: Proceeding of First Chilean Workshop on Pattern Recognition: Theory and Applications, pp. 44-51 (2009)

19. Hari, V.: Accelerating the SVD Block-Jacobi Method. Computing 75, 27-53 (2005)

20. Blumenstein, M., Ferrer Miguel, A., Vargas, J.F.: The 4NSigComp2010 off-line signature verification competition: Scenario 2. In: Proceedings of 12 th International Conference on Frontiers in Handwriting Recognition, Kolkata, India, November 16-18, pp. 721-726 (2010) ISSBN: 978-0-7695-4221-8 